\title{
THE GLUTAMATE ANALOGUE $\alpha$-AMINOADIPIC ACID IS TAKEN UP BY ASTROCYTES BEFORE EXERTING ITS GLIOTOXIC EFFECT IN $\operatorname{VITRO}^{1}$
}

\author{
SIGISMUND HUCK, ${ }^{* 2}$ FRIEDRICH GRASS, ${ }^{*}$ AND HEIDE HÖRTNAGL $\ddagger$ \\ Departments of ${ }^{*}$ Neuropharmacology and $\ddagger$ Biochemical Pharmacology, University of Vienna, A-1090 Vienna, Austria
}

Received March 5, 1984; Revised May 8, 1984; Accepted May 16, 1984

\begin{abstract}
DL- and L- $\alpha$-aminoadipic acid ( $\alpha$-AA) are specific gliotoxins in vitro (Huck, S., F. Grass, and M. E. Hatten (1984) Neuroscience 12: 783-796). By combining immunohistochemical and autoradiographic techniques, we now show that DL- $\left[{ }^{14} \mathrm{C}\right]-\alpha-\mathrm{AA}$ is accumulated almost selectively by astrocytes in cultures of the dissociated postnatal mouse cerebellum, presumptive neurons being free of the radiolabel. High pressure liquid chromatography analysis of cultures incubated with D- or L- $\alpha$-AA and DL- $\left[{ }^{14} \mathrm{C}\right]-\alpha$-AA autoradiograms conducted in the presence of $\mathrm{D}$ - or $\mathrm{L}-\alpha$-AA reveal a stereospecificity of astroglial $\mathrm{L}-\alpha$-AA uptake.

Both the uptake of $\alpha$-AA by astrocytes and $\alpha$-AA-induced gliotoxicity were sodium dependent. Since $2 \mu \mathrm{M}$ tetrodotoxin did not prevent the morphological changes, we conclude that sodium plays its role in $\alpha$-AAinduced gliotoxicity by mediating the transport of the substance.Thus, $\alpha$-AA appears to be taken up by the astrocytes before exerting its cytotoxic effect.
\end{abstract}

$\alpha$-Aminoadipic acid ( $\alpha$-AA), a six-carbon homologue of glutamate, is a specific gliotoxic agent in vivo (Olney et al., 1971, 1980) and in vitro (Casper et al., 1982). We have recently confirmed this glial-specific effect in cultures of the dissociated postnatal mouse cerebellum (Huck et al., 1984). We reported that DL- and L- $\alpha$-AA, while sparing neurons, caused the cell death of astrocytes in our culture system. D- $\alpha-\mathrm{AA}$, on the other hand, was only slightly effective.

The mechanism underlying the $\alpha$-AA-induced gliotoxicity is not known. However, it is well documented that astrocytes are equipped with an extremely potent $\mathrm{Na}^{+}$-dependent carrier system for glutamate (Faivre-Bauman et al., 1974; Henn et. al., 1974; Balcar et. al., 1977; Schousboe et al., 1977; Drejer et al., 1982; Gordon and Balázs, 1983). This system has been shown to transport not only L-glutamate, but also related amino acids like D- and L-aspartate (Balcar et al., 1977; Schousboe et al., 1977; Drejer et al., 1982; Gordon and Balázs, 1983). Consequently, it might also transport $\alpha$-AA as a close structural homologue of glutamate.

Thus, this study was designed to seek a sodium-dependent transport of $\alpha$-AA by astrocytes in vitro. In addition, we wished to know whether this transport would agree with our previous observation concerning the stereospecificity of the $\alpha$-AA effects. Finally, we investigated whether a block of the $\alpha$-AA

\footnotetext{
${ }^{1}$ Supported by Austrian Scientific Research Fund Projects S25/02 and S25/05. The assistance of Mrs. A. Motejlek and Mr. H. Reither is gratefully acknowledged. We thank Dr. A. Ellinger for his help in setting up autoradiography. The AbGFA was a generous gift of Dr. E. Bock, Copenhagen.

${ }^{2}$ To whom correspondence should be addressed.
}

transport by omitting $\mathrm{Na}^{+}$from the incubation medium would also prevent $\alpha$-AA-induced gliotoxic effects.

\section{Materials and Methods}

Cell cultures. The cerebellar tissue of 7-day-old HimOF1(Swiss)SPF mice was removed by dissection, and single-cell suspensions were prepared as described (Trenkner and Sidman, 1977; Hatten and Sidman, 1978; Huck, 1983). Cells were plated in the absence of serum at a density of 1 to $1.5 \times 10^{6} / \mathrm{ml}$ in $3.5-\mathrm{cm}(2-\mathrm{ml}$ cell suspension) tissue culture dishes (Nunc, catalog no. 153060) or 24-multiwell $(0.4 \mathrm{ml} /$ well) tissue culture plates (Nunc, catalog no. 168357). All microscopic observations were made with cells grown on glass coverslips (Menzel, no. 1, $12-\mathrm{mm}$ diameter) which were placed in the culture dish or mounted as a false bottom over a $5-\mathrm{mm}$ hole in the dish as described (Huck and Hatten, 1981). The culture medium consisted of Eagle's basal medium supplemented with $2 \mathrm{mM}$ glutamine, $25 \mathrm{units} / \mathrm{ml}$ of penicillin-streptomycin, $17 \mathrm{~mm}$ glucose, and $31 \mu \mathrm{g} / \mathrm{ml}$ of insulin, except for a short exposure of the cells to $10 \%$ horse serum to improve viability of the cultures (Huck et al., 1984). This technique yields cultures with plating efficiencies of approximately $60 \%$ after 1 week of incubation.

All experimental incubations were performed in HEPES-buffered media with the $\mathrm{pH}$ adjusted to 7.4 (at room temperature). The $\mathrm{Na}^{+}$. containing medium consisted of (millimolar): $\mathrm{Na}^{+}(137.5), \mathrm{K}^{+}(5.77)$, $\mathrm{Ca}^{2+}(1.26), \mathrm{Mg}^{2+}(0.89), \mathrm{Cl}^{-}(138.0), \mathrm{HSO}_{4}^{-}(0.4), \mathrm{HPO}_{4}^{-}(0.77)$, and HEPES $(10.0)$. In the sodium-"free" medium, all $\mathrm{Na}^{+}$except $3 \mathrm{~mm}$ was replaced by the appropriate amount of choline. The $\mathrm{Ca}^{2+}$-free medium was prepared by substituting $\mathrm{Ca}^{2+}$ with the appropriate amount of $\mathrm{Na}^{+}$. All media contained 25 units $/ \mathrm{ml}$ of penicillin-streptomycin, $17 \mathrm{mM}$ glucose, and vitamins and amino acids in the concentrations recommended by Eagle (1955) but no glutamine.

Immunohistochemistry. Astrocytes were identified by indirect immunofluorescence staining of glial fibrillary acidic protein (GFA). The antisera and fixation and staining procedures were identical to those in a previous study (Huck, 1983), except that cultures were fixed with 
$4 \%$ paraformaldehyde (in $0.1 \mathrm{M}$ cacodylate, $\mathrm{pH} 7.4$ ) for $2 \mathrm{hr}$ and permeabilized by $0.2 \%$ Triton $\mathrm{X}-100$ (in $4 \%$ paraformaldehyde and 0.1 M cacodylate, $\mathrm{pH} 7.4$ ) for 5 min when immunohistochemistry was combined with autoradiography.

Autoradiography. Cultures grown on $12-\mathrm{mm}$ glass coverslips were used after 5 to 6 days in vitro. Prior to experimental incubations cultures were transferred to HEPES-buffered media of indicated ionic compositions. Incubations with DL- $\left[6-{ }^{14} \mathrm{C}\right]-\alpha$-AA (Commissariat à l'Energie Atomique (France), specific activity: $46 \mathrm{mCi} / \mathrm{mmol}, 1 \mu \mathrm{Ci} / \mathrm{ml}$, actual concentration: $21 \mu \mathrm{M}$ ) or L-[ $\left[\mathrm{G}^{3} \mathrm{H}\right]$ glutamate (New England Nuclear, specific activity: $35 \mathrm{Ci} / \mathrm{mmol}, 1 \mu \mathrm{Ci} / \mathrm{ml}$, actual concentration: 28 $\mathrm{nM}$ ) were conducted at room temperature. In general, cultures were incubated with $\mathrm{DL}-\left[{ }^{14} \mathrm{C}\right]-\alpha-\mathrm{AA}$ for $10 \mathrm{~min}$ or with $\mathrm{L}-\left[{ }^{3} \mathrm{H}\right]$ glutamate for $5 \mathrm{~min}$, washed in PBS for $2 \mathrm{~min}$, and fixed in $2.5 \%$ glutaraldehyde (in $0.2 \mathrm{M}$ cacodylate, $\mathrm{pH} 7.4$ ) for $2 \mathrm{hr}$. For combined immunohistochemistry and autoradiography, the incubation time was $15 \mathrm{~min}$, and cultures were fixed with paraformaldehyde since glutaraldehyde fixation was not compatible with GFA immunohistochemistry (S. Huck, unpublished observation).

After a brief rinse in distilled water, cultures were allowed to air dry, fastened to glass slides, dipped into Kodak NTB-2 nuclear track emulsion (diluted 1:2.5 with distilled water and heated to $37^{\circ}$ ), and exposed for 4 to 7 days in the cold room. Developing of the photoemulsion (Kodak D-19) and fixation were by conventional techniques. Finally, cultures were mounted in glycerin-PBS (1:1) and viewed by brightfield, phase contrast, differential interference contrast (Nomarski), or fluorescence optics.

Release of radioactivity from cultures by osmotic shock. Experiments to study the release of accumulated $\mathrm{DL}-\left[{ }^{14} \mathrm{C}\right]-\alpha$-AA into isotonic (290 $\mathrm{mM}$ sucrose) or hypotonic (double-distilled water) supernatants were performed on cultures grown in 24-multiwell plates for 5 days. Cultures loaded by a 10 - $\mathrm{min}$ incubation with $\mathrm{DL}-\left[{ }^{14} \mathrm{C}\right]-\alpha-\mathrm{AA}(1 \mu \mathrm{Ci} / \mathrm{ml})$ at room temperature were washed three times for $1 \mathrm{~min}$ with $\mathrm{PBS}$ and incubated with either isotonic sucrose $(290 \mathrm{mM})$ for $10 \mathrm{~min}$ or double-distilled water for $2 \mathrm{~min}$. The amount of radioactivity released into the supernatants was determined before and after passing them through immersible $10,000-\mathrm{mW}$ cutoff filter units (Millipore, CX-10). Less than 10 min were required to recover quantities of ultrafiltrate sufficient for liquid scintillation counting. Since the cells readily detached from the substratum when subjected to osmotic shock, the incubation of cultures with sucrose was $10 \mathrm{~min}$ as compared to 2 min with $\mathrm{H}_{2} \mathrm{O}$ in order to provide equal chances for DL- $\left[{ }^{14} \mathrm{C}\right]-\alpha$-AA to dissociate from membranes. The radioactivity retained by the cultures was determined, after dissolving the cells in $2 \mathrm{~N} \mathrm{KOH}$, by adding $400 \mu$ l of neutralized aliquots to $5 \mathrm{ml}$ of Atomlight (New England Nuclear) scintillation solution.

High pressure liquid chromatography (HPLC) determination of $\alpha-A A$ uptake. Prior to experimental incubations, cultures grown in $35-\mathrm{mm}$ tissue culture dishes for 5 to 6 days in vitro were washed twice with sodium-containing or sodium-free HEPES-buffered medium. Incubations with $\mathrm{D}$ - or $\mathrm{L}-\alpha$-AA were conducted in $\mathrm{Na}^{+}$-containing or $\mathrm{Na}^{+}$-free HEPES-buffered medium for $4 \mathrm{hr}$ at $35.5^{\circ} \mathrm{C}$. Thereafter, cultures were washed twice with ice-cold PBS, harvested in $2 \mathrm{ml}$ of distilled $\mathrm{H}_{2} \mathrm{O}$ (with the material of five dishes pooled for one HPLC determination), ultrasonicated, and centrifuged at $10,000 \times g$. Ten microliters of the supernatant were injected into a HPLC system which was set up according to Schmid et al. (1980), except that the column $(0.3 \times 27$ $\mathrm{cm}$ ) was packed with Bio-Rad Aminex HP-C cation exchange resin, maintained at $76^{\circ} \mathrm{C}$ with electrical heating tape. In this system $\alpha$-AA eluted well separated between glutamic acid and glycine.

\section{Results}

Approximately $5 \%$ of the cells in the autoradiograms were strongly labeled, following incubation of the cerebellar cultures with DL- $\left[{ }^{14} \mathrm{C}\right]-\alpha-\Lambda \Lambda$. These cells were not phase bright, had thick processes, and were probably glial in origin (Huck et al., $1984)$. Silver grains were located over the perikaryal region as well as over the major processes (Figs. $1, A$ and $B$, and $2 B$ ). In contrast few, if any, silver grains were seen over the small, much more numerous cells which were phase bright, a characteristic feature of neurons in vitro (Figs. $1 A$ and $2 B$ ).

A similar pattern of radiolabeling was observed after incubating the cultures with $\mathrm{L}-\left[{ }^{3} \mathrm{H}\right]$ glutamate, except that silver grain accumulation more closely followed the outlines of astrocytic cell bodies and processes (Fig. 2A).

Substitution of choline for sodium in the incubation medium to inhibit $\mathrm{Na}^{+}$-dependent uptake processes prevented the DL$\left[{ }^{14} \mathrm{C}\right]-\alpha$-AA labeling of astrocytes (Fig. $1, C$ and $D$ ). Likewise, the density of silver grains over astrocytes was markedly reduced when cultures were treated with $5 \mathrm{mM}$ ouabain $30 \mathrm{~min}$ before and during the experimental incubation with $\mathrm{DL}-\left[{ }^{14} \mathrm{C}\right]-$ $\alpha$-AA (not shown).

The presence of $5 \mathrm{mM} \mathrm{L}-\alpha-\mathrm{AA}$ (Figs. $1, E$ and $F$ ), $5 \mathrm{mM} \mathrm{L}$ glutamate, or $5 \mathrm{mM} \mathrm{L}$ - and D-aspartate in the incubation medium prevented the accumulation of silver grains over astrocytes. In contrast, $5 \mathrm{mM} \mathrm{D}-\alpha$-AA (Fig. $1, A$ and $B$ ), $5 \mathrm{~mm} N$ methyl-D-aspartate, or $5 \mathrm{mM} \alpha$-amino-4-phosphonobutyric acid had no effect on the autoradiographic localization of DL- $\left[{ }^{14} \mathrm{C}\right]-$ $\alpha$-AA.

To confirm the characterization of cell types made by phase contrast microscopy, we combined DL- $\left[{ }^{14} \mathrm{C}\right]-\alpha$-AA autoradiography with immunocytochemical localization of antiserum raised against human glial fibrillary acidic protein (AbGFA), a marker of astrocytes. All AbGFA-positive cells accumulated the radiolabel (Fig. 3), even though the silver grain density over individual cells varied considerably. In contrast to astrocytes, silver grains were not above background over presumptive neurons (Fig. 3). On the other hand, some flattened, AbGFAnegative cells accumulated small amounts of the radiolabel (not shown).

Shock release of internalized $D L-\left[{ }^{4} C\right]-\alpha-A A$. Cultures that had been loaded with DL- $\left[{ }^{14} \mathrm{C}\right]-\alpha$-AA released some activity when incubated in an isotonic medium for $10 \mathrm{~min}$ but almost all of the radiolabel when subjected to a brief osmotic shock (Table I). This activity passed an immersible $10,000-\mathrm{mW}$ cutoff filter unit and was, therefore, considered unbound by membranes or proteins (Table I)

$H P L C$-measured $\alpha-A A$ uptake. Cells harvested after a $4-\mathrm{hr}$ incubation with $42 \mu \mathrm{M} \mathrm{L}-\alpha$-AA retained approximately $50 \%$ of the substance that had initially been added to the culture medium (Table II). No cellular accumulation of $\mathrm{L}-\alpha-\mathrm{AA}$ was detected hy HPLC when choline was substituted for $\mathrm{Na}^{+}$in the incubation medium. On the other hand, cultures retained measurable amounts of D- $\alpha$-AA only when incubated at $436 \mu \mathrm{M}$ but not at $37 \mu \mathrm{M}$. Again, the accumulation of $\mathrm{D}-\alpha-\mathrm{AA}$ was sodium dependent (Table II).

$N a$ dependency of $\alpha-A A$ effects. Addition of DL- or L- $\alpha$-AA to sodium-containing incubation medium leads to rapid morphological changes of astrocytes (nuclear and cytoplasmic swelling, karyopyknosis) (Huck et al., 1984). These $\alpha$-AA-induced changes were prevented when choline was substituted for sodium in the incubation medium (Fig. 4).

Tetrodotoxin $(2 \mu \mathrm{M})$ or tetracaine $(0.1 \mathrm{mM})$ did not block the gliotoxic effect of $\alpha$-AA. Likewise, the $\alpha$-AA-induced changes in astrocytes were not prevented by using $\mathrm{a} \mathrm{Ca}^{2+}$-free incubation medium.

\section{Discussion}

This autoradiographic study demonstrates a sodium-dependent accumulation of $\mathrm{DL}-\left[{ }^{14} \mathrm{C}\right]-\alpha-\mathrm{AA}$ by astrocytes in vitro. Since the radioactivity is readily released from the cultures by osmotic shock, this accumulation appears to be uptake rather than binding of $\alpha$-AA to the outside of the cell membrane.

All AbGFA-stained (Antanitus et al., 1975), presumptive astrocytes in the culture accumulated $D L-\left[{ }^{14} \mathrm{C}\right]-\alpha$-AA, even though the density of silver grains over individual astrocytes varied considerably. This may reflect differences in the transport kinetics of individual cells, differences in the metabolic rates, or differential losses of the radiolabel during the fixation with paraformaldehyde that were considerable. A similar loss 

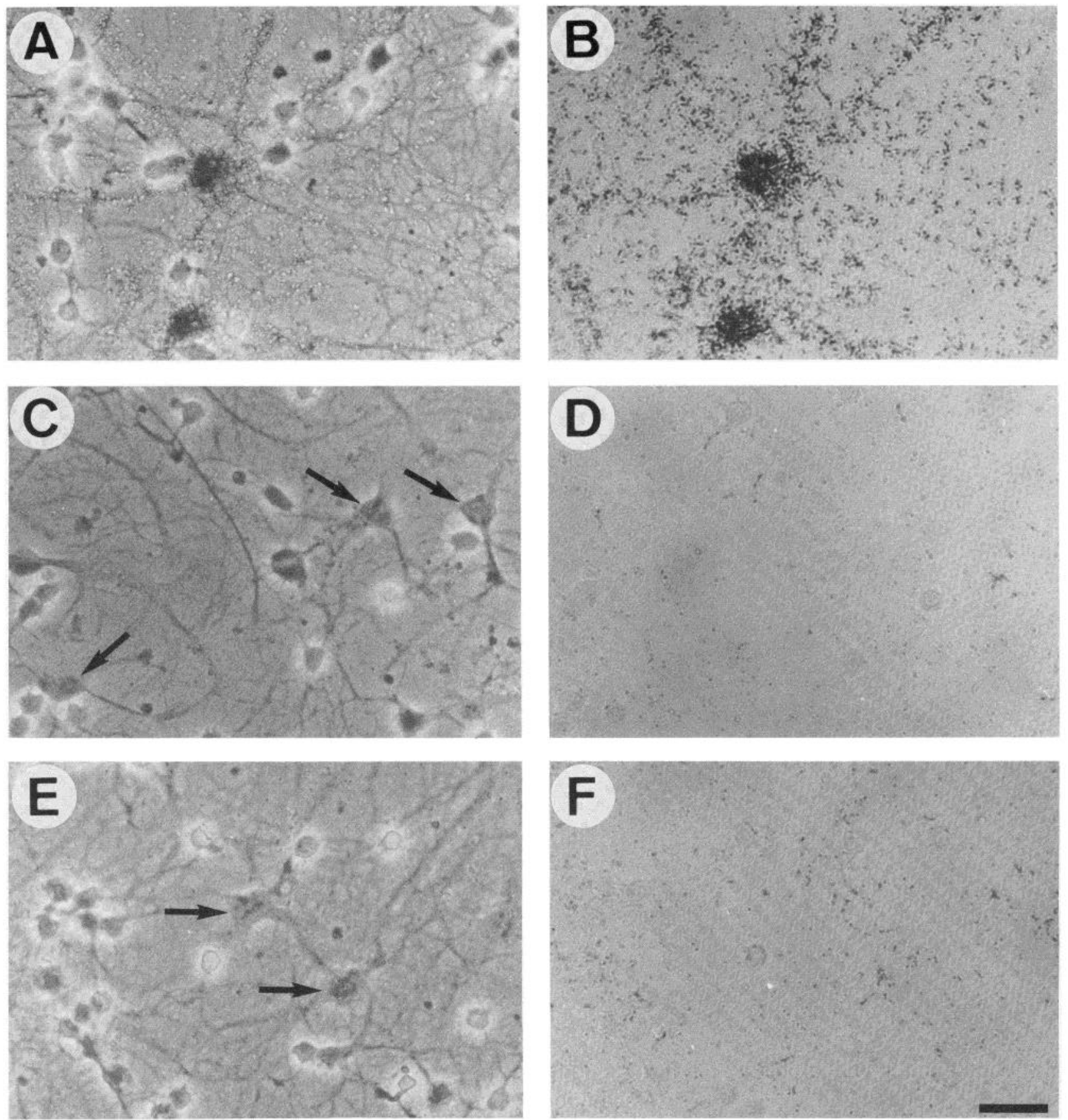

Figure 1. Corresponding phase contrast and brightfield pictures of DL- $\left[{ }^{14} \mathrm{C}\right]-\alpha$-AA autoradiograms. $A$ and $B$, Experimental incubation in sodium-containing, HEPES-buffered medium in the presence of $5 \mathrm{mM}$ D- $\alpha$-AA. Silver grain density corresponds with autoradiograms of cultures incubated in the absence of D- $\alpha$-AA (see Fig. $2 B$ ). $C$ and $D$, Experimental incubation in sodium-"free," HEPES-buffered medium. Presumptive astrocytes (arrows) fail to accumulate significant amounts of the radiolabel. $E$ and $F$, Experimental incubation in sodium-containing, HEPESbuffered medium in the presence of $5 \mathrm{mM} \mathrm{L}-\alpha-\mathrm{AA}$. Uptake of DL- $\left[{ }^{14} \mathrm{C}\right]-\alpha-\mathrm{AA}$ is almost totally prevented by the unlabeled $\mathrm{L}-\alpha-\mathrm{AA}$. Presumptive astrocytes are indicated by arrows. Calibration bar, $20 \mu \mathrm{m}$.

of $\left[{ }^{3} \mathrm{H}\right] \mathrm{GABA}$ from cultures fixed with paraformaldehyde has recently been reported (Neale et al., 1983; Hatten et al., 1984). In these studies glutaraldehyde also interfered with immunohistochemistry and could not be used when autoradiography was combined with immunohistochemistry.

Whereas some flat, GFA-negative non-neuronal cells accumulated small amounts of $\mathrm{DL}-\left[{ }^{14} \mathrm{C}\right]-\alpha$-AA, presumptive neurons appeared free of the label. This absence of $\alpha$-AA uptake is not related to reduced size of the neurons, since in autoradiograms following the incubation of cultures with $\left[{ }^{3} \mathrm{H}\right] \mathrm{GABA}$, even small neurons may appear heavily labeled (S. Huck, unpublished observation). However, our data cannot exclude the possibility of an $\alpha$-AA uptake into the fine processes, if not into the perikaryon, of neurons.

The autoradiograms obtained with DL- $\left[{ }^{14} \mathrm{C}\right]-\alpha$-AA essentially matched those obtained from cultures incubated with $\mathrm{L}-\left[{ }^{3} \mathrm{H}\right]$ glutamate, except that in the latter case silver grain accumulation followed more closely the outlines of cell body and processes of astrocytes, possibly because of the shorter range of ${ }^{3} \mathrm{H}$. These findings are consistent with previous autoradi- 

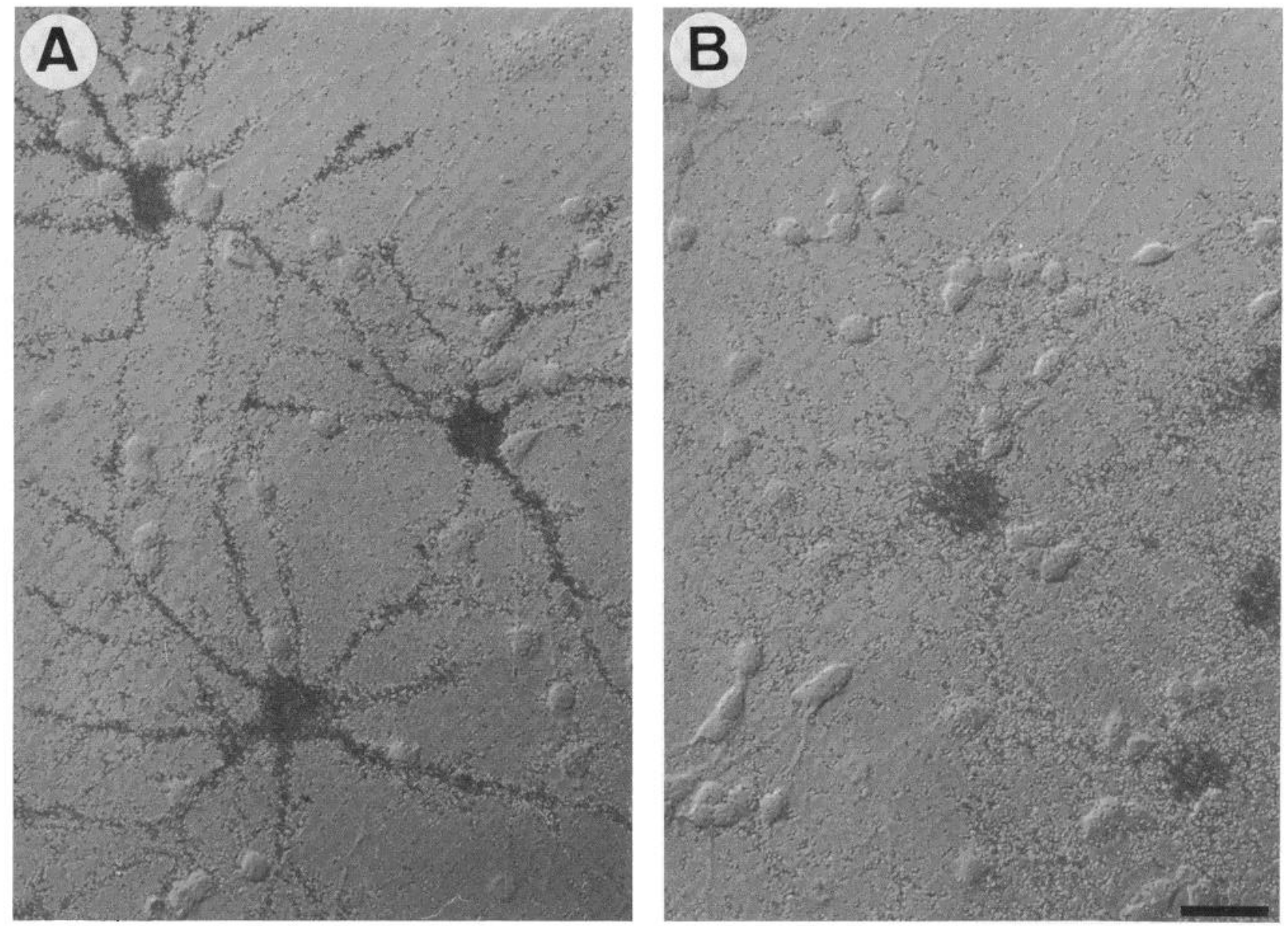

Figure 2. Autoradiograms of cerebellar cultures following incubation with $28 \mathrm{nM} \mathrm{L}-\left[{ }^{3} \mathrm{H}\right]$ glutamate $(A)$ or $21 \mu \mathrm{M}$ DL- $\left[{ }^{14} \mathrm{C}\right]-\alpha-\mathrm{AA}(B)$. Silver grain accumulation, apparently restricted to astrocyte-like cells, follows more closely the outlines of cell body and processes in the case of $L-\left[{ }^{3} \mathrm{H}\right]$ glutamate incubation, presumably because of the shorter range of ${ }^{3} \mathrm{H}$. Nomarski optics. Calibration bar, $20 \mu \mathrm{m}$.
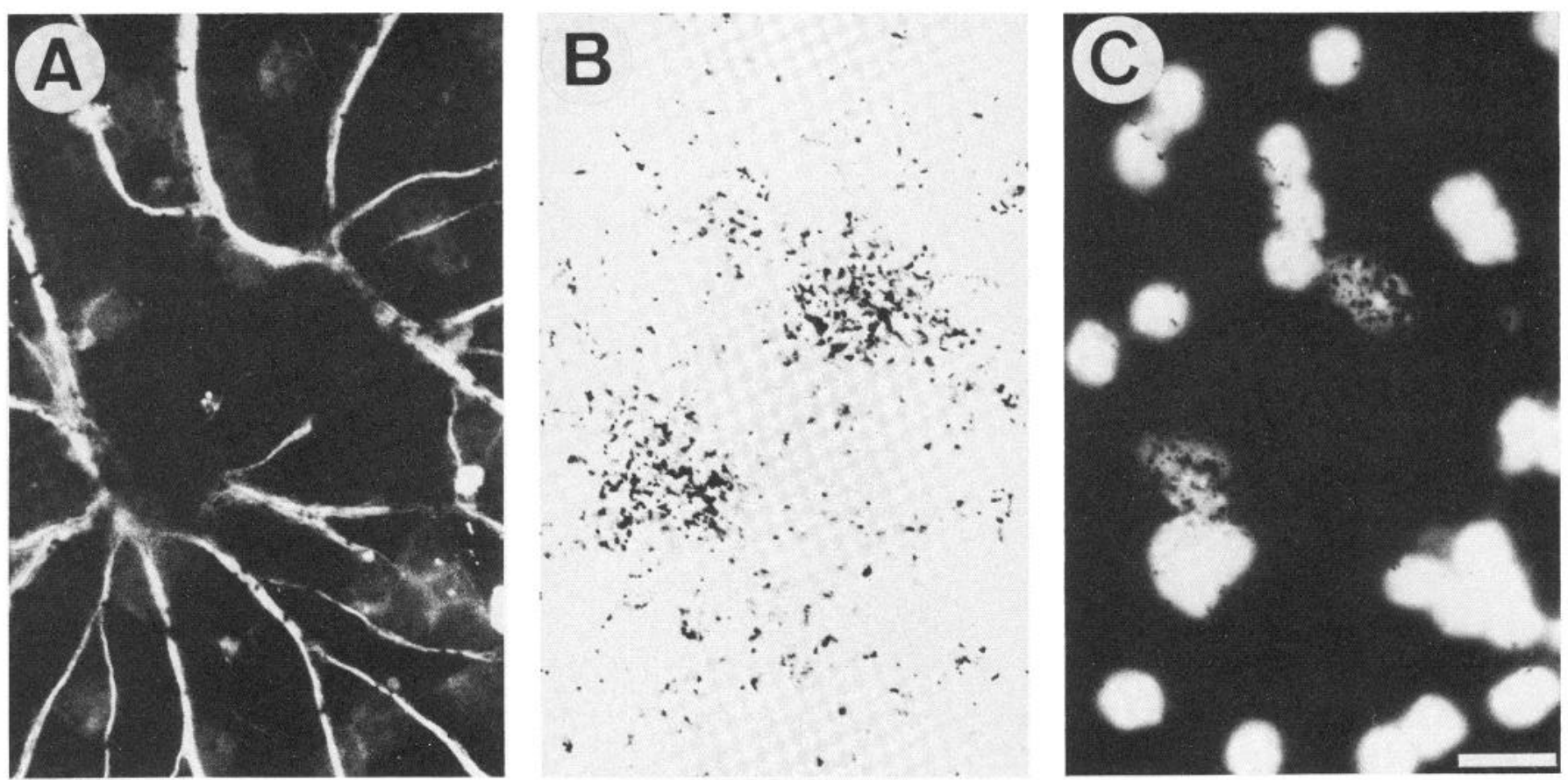

Figure 3. Combined GFA immunohistochemistry and DL- $\left[{ }^{14} \mathrm{C}\right]-\alpha$-AA autoradiography. Both the brightfield picture $(B)$ and the fluorescence staining with Nuclear Yellow $(C)$ show two moderately labeled astrocytes which were identified by indirect immunofluorescence staining using rabbit anti-human GFA antiserum (1:50) and rhodamine-labeled goat anti-rabbit IgG $(1: 100)(A)$. Close to these cells, astrocytes with both higher and lower silver grain densities were located. Note that the silver grain density is significantly reduced in these paraformaldehyde-fixed cultures as compared to glutaraldehyde-fixed cells (Figs. 1 and 2). Calibration bar, $20 \mu \mathrm{m}$. 
ographic (de Barry et al., 1982; Wilkin et al., 1982; Gordon and Balázs, 1983; Levi et al., 1983) and kinetic (Gordon and Balázs, 1983) studies showing a preferential uptake of $\mathrm{L}-\left[{ }^{3} \mathrm{H}\right]$ glutamate or $\mathrm{D}-\left[{ }^{3} \mathrm{H}\right]$ aspartate by cerehellar astrocytes.

The properties of the high affinity uptake system of glutamate have been studied in some detail in various preparations (Balcar and Johnston, 1972; Logan and Snyder, 1972; Bennet et al., 1973; Henn et al., 1974; Roberts and Watkins, 1975; Balcar et al., 1977; Stallcup et al., 1979; Vincent and McGeer, 1980; Drejer et al., 1982; Gordon and Baläzs, 1983). This uptake has been shown to be largely sodium dependent (Balcar and Johnston, 1972; Bennet et al., 1972, 1973; Faivre-Bauman et al., 1974; Henn et al., 1974; Balcar et al., 1977; Stallcup et al., 1979; Wheeler, 1979; Drejer et al., 1982; Gordon and Balázs, 1983) and to transport several acidic amino acids with relatively little specificity (Balcar and Johnston, 1972; Logan and Snyder, 1972; Bennet et al., 1973; Drejer et al., 1982; Gordon and Balázs, 1983). Therefore, this acidic amino acid carrier system may transport also $\alpha$-AA as a close structural homologue of glutamate. The reportedly low potency of $\alpha$-AA to inhibit high affinity glutamate uptake is not inconsistent with this hypothesis, especially since most previous investigations used the racemic mixture of $\alpha$-AA (Balcar and Johnston, 1972; Schousboe et al., 1977; Vincent and McGeer, 1980; Charles and Chang, 1981; Drejer et al., 1982). L- $\alpha$-AA, which is taken up by the cultures much better than $\mathrm{D}-\alpha-\mathrm{AA}$, should also compete more effectively than DL- $\alpha$-AA with the other amino acids transported by this system. The stereospecificity of the $\alpha$-AA transport, as demonstrated in this study by HPLC, is also reflected

\section{TABLE I}

Release of accumulated $D L-\left[{ }^{14} C\right]-\alpha-A A$ from cultures into isotonic (290 mM sucrose) or hypotonic (double-distilled water) supernatants Cerebellar cells were plated at a density of $5.4 \times 10^{5} / 0.4 \mathrm{ml} /$ well in Nunc 24-well tissue culture cluster dishes. For either approach, the material of six cultures was pooled. Numbers are counts per minute per culture.

\begin{tabular}{lcccc}
\hline & Total & Culture & Supernatant & Filtrate $^{\alpha}$ \\
\hline Sucrose & $6451(100 \%)$ & $5443(84 \%)$ & $1008(16 \%)$ & 1065 \\
Water & $6129(100 \%)$ & $298(5 \%)$ & $5831(95 \%)$ & 5650 \\
\hline
\end{tabular}

${ }^{a}$ Supernatants after being passed through an immersible 10,000 $\mathrm{mW}$ cutoff filter unit. by our data based on $\mathrm{DL}-\left[{ }^{14} \mathrm{C}\right]-\alpha$-AA autoradiography. Whereas L- $\alpha$-AA significantly inhibited silver grain accumulation over astrocytes, D- $\alpha$-AA was without noticeable effect. These results are in agreement with a recent study showing preferential uptake of $\mathrm{L}-\alpha$-AA into rat cortical slices (Charles and Chang, 1981). Thus, the stereospecificity of the $\alpha$-AA transport parallels previous observations that L- $\alpha$-AA has a significantly higher gliotoxic potency than D- $\alpha$-AA (Olney et al., 1980; Huck et al., 1984).

According to the autoradiographic data presented in this study, not only L- $\alpha$-AA but also $\mathrm{L}$-glutamate and $\mathrm{L}$ - and $\mathrm{D}$ aspartate prevent the accumulation of $\mathrm{DL}-\left[{ }^{14} \mathrm{C}\right]-\alpha-\mathrm{AA}$ into astrocytes. These results are in line with a recent study showing inhibition of $\mathbf{L}-\left[{ }^{3} \mathrm{H}\right]-\alpha$-AA uptake into rat cortical slices by $L$ glutamate and L-aspartate (Charles and Chang, 1981) and are to be expected if $\mathrm{L}-\alpha$-AA shares a carrier in common with $\mathrm{L}$ glutamate and L-aspartate. Nevertheless, detailed kinetic data are clearly required before regarding $\mathrm{L}-\alpha$-AA, together with $\mathrm{D}$ and $\mathrm{L}$-aspartate, as a substratum for the high affinity glutamate transport system.

$\alpha$-AA strictly required the presence of sodium in the incubation medium to induce morphological changes and cell death of astrocytes. This cytotoxic effect is apparently unrelated to voltage-dependent sodium channels known to be present in cultured astrocytes (Nowak et al., 1983; Reisner et al., 1983). At the present, we cannot exclude the possibility that $\alpha$-AA might open tetrodotoxin-resistant sodium channels in astrocytes by a mechanism similar to the long-lasting depolarization of neurons in response to glutamate (Puil, 1981). On the other hand, the results presented in this study demonstrate the importance of $\mathrm{Na}^{+}$for the accumulation of $\alpha$-AA by astrocytes and suggest that sodium plays its role in $\alpha$-AA-induced gliotoxicity by mediating the uptake of the substance.

This pathophysiological mechanism would be entirely different from the proposed mode of action of neurotoxic amino acids, which most likely act by the outer membrane of the neuron (Kizer et al., 1978). By using a relatively simple glutamatergic system, the neuromuscular junction of the locust leg, recent evidence suggests $\mathrm{Ca}^{2+}$ as a mediator of glutamateinduced myopathy (Duce et al., 1983), which was significantly slowed in $\mathrm{Ca}^{2+}$-free saline. In contrast, the morphological response of astrocytes to $\alpha$-AA appeared unaffected when cultures were incubated $\mathrm{Ca}^{2+}$-free, indicating a further difference be-

\section{TABLE II}

$H P L C$-measured $\alpha-A A$ uptake

Cerebellar cells were plated at a density of $2.8 \times 10^{6} / 2 \mathrm{ml} /$ dish. After 5 days in vitro, cultures were incubated with D- or L- $\alpha$-AA in the presence $\left(+\mathrm{Na}^{+}\right)$or absence $\left(-\mathrm{Na}^{+}\right)$of sodium for $4 \mathrm{hr}$. For each HPLC analysis, the material from five tissue culture dishes (35-mm diameter) was pooled.

\begin{tabular}{|c|c|c|c|}
\hline \multirow{2}{*}{ Stereoisomer } & \multicolumn{3}{|c|}{$\alpha$-AA per Five Dishes } \\
\hline & Offered $^{a}$ & Culture $^{b}$ & Supernatant \\
\hline & \multicolumn{3}{|c|}{$\mu \mathrm{mol}$} \\
\hline $42 \mu \mathrm{ML}-\alpha-\mathrm{AA}+\mathrm{Na}^{+}\left(\mathrm{n}=6^{d}\right)$ & 0.21 & $0.103 \pm 0.014\left(49 \%^{e}\right)$ & $0.095 \pm 0.022(45 \%)$ \\
\hline $39 \mu \mathrm{M} \mathrm{L}-\alpha-\mathrm{AA}-\mathrm{Na}^{+}(\mathrm{n}=3)$ & 0.19 & $0^{f}$ & $\mathrm{ND}^{\mathrm{g}}$ \\
\hline $37 \mu \mathrm{M}$ D- $\alpha-\mathrm{AA}+\mathrm{Na}^{+}(\mathrm{n}=3)$ & 0.18 & $0^{f}$ & ND \\
\hline $436 \mu \mathrm{M}$ D- $\alpha-\mathrm{AA}+\mathrm{Na}^{+}(\mathrm{n}=3)$ & 2.18 & $0.065 \pm 0.004(3 \%)$ & ND \\
\hline $401 \mu \mathrm{M}$ D- $\alpha-\mathrm{AA}-\mathrm{Na}^{+}(\mathrm{n}=3)$ & 2.00 & $0^{f}$ & ND \\
\hline
\end{tabular}

${ }^{a}$ Amount of starting material per five cultures (micromoles).

${ }^{b}$ The amount of $\alpha$-AA accumulated by the cells. Numbers are micromoles of $\alpha$-AA per five dishes (mean \pm SD, calculated from data of individual HPLC determinations). Since the material of five cultures was pooled for one HPLC determination, $6 \times 5$ cultures (first row) or $3 \times$ 5 cultures (second to fifth rows) were used for this experiment.

${ }^{c}$ Numbers represent micromoles of $\alpha$-AA per five dishes (mean $\pm \mathrm{SD}$ ) recovered from the incubation medium.

${ }^{d} n$, Number of HPLC determinations.

${ }^{e}$ The numbers in parentheses are the amounts of $\alpha$-AA, expressed as a percentage of the starting material. Note that in spite of the considerable uptake of $\mathbf{L}-\alpha$-AA, more than $90 \%$ of the starting material was recovered as native $\alpha$-AA.

${ }^{f}$ Less than $0.002 \mu \mathrm{mol}$ present in the material.

${ }^{8} \mathrm{ND}$, Not determined. 

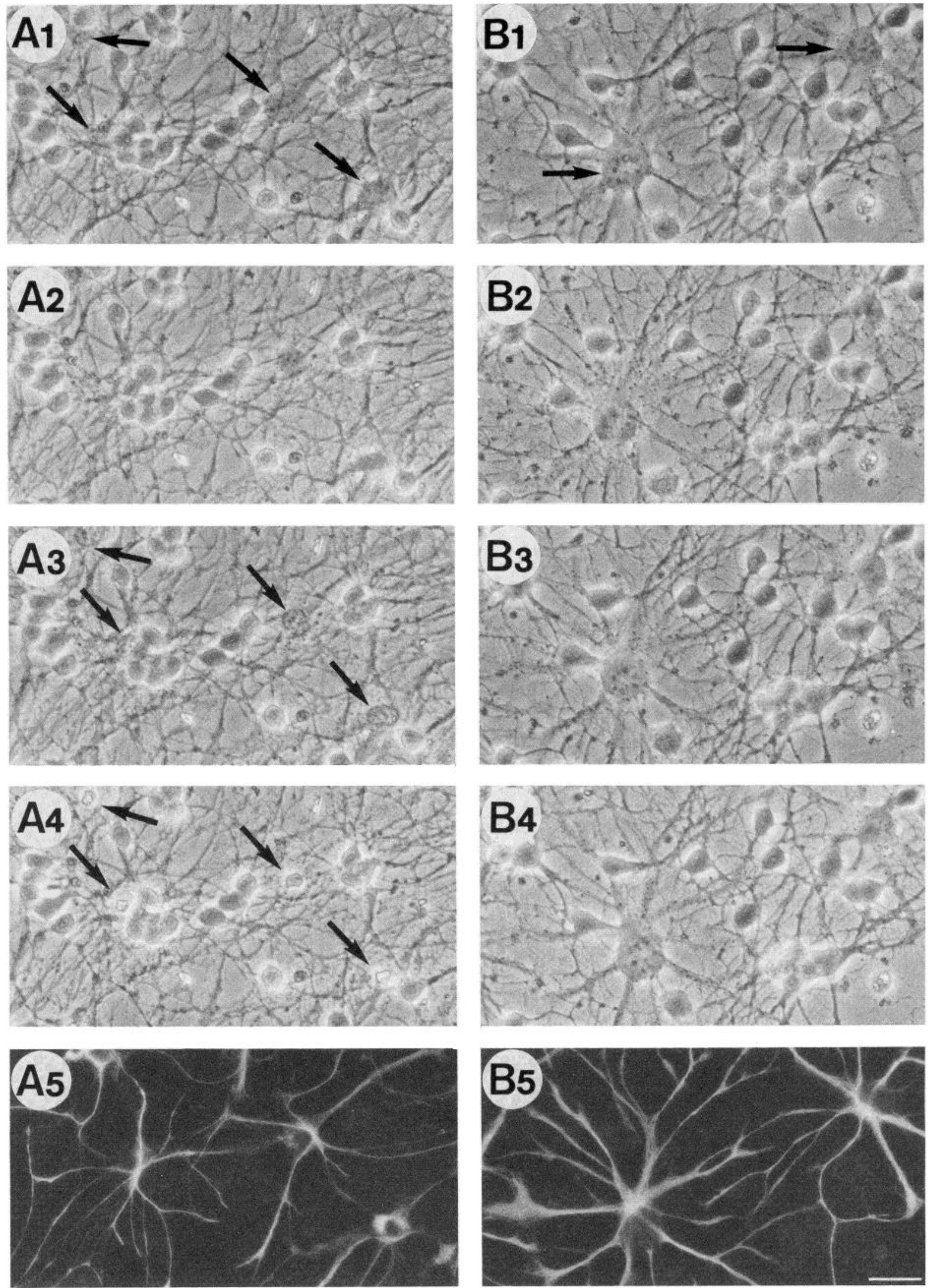

Figure 4. Sodium dependency of $\alpha$-AA-induced gliotoxic effects. Two cerebellar cultures $(A$ and $B)$ were first incubated in sodium-free, HEPES-buffered medium $(A 1$ and $B 1)$ in the presence of $0.34 \mathrm{mM} \mathrm{L}-\alpha$-AA (A2 and $B 2: 3 \mathrm{hr}$ after adding the agent). Incubation conditions were then changed to sodium-containing, HEPES-buffered medium with $0.34 \mathrm{~mm} \mathrm{~L}$ - $\alpha$-AA being present in $A$ ( $A 3: 30 \mathrm{~min} ; A 4: 3 \mathrm{hr}$ ), but not in $B$ ( $B 3$ and $B 4: 30 \mathrm{~min}$ and $3 \mathrm{hr}$ after the medium change, respectively). Arrows in $A 1$ and $B 1$ indicate astrocytes according to the immunofluorescence pictures shown in $A 5$ and B5. Note that pronounced morphological changes of astrocytes (arrows) shown in $A 3$ and $A 4$ are induced only if L- $\alpha$ $\mathrm{AA}$ and $\mathrm{Na}^{+}$are simultaneously present in the incubation medium. Calibration bar, $20 \mu \mathrm{m}$. 
tween the mechanism of $\alpha$-AA-induced gliotoxicity and the neurotoxic effects of excitatory amino acids.

$\alpha$-AA is a natural product of lysine metabolism in the brain (Chang, 1978, 1982). However, in spite of its natural occurrence and its rapid clearance from the brain in vivo (Chang, 1978), the metabolism of $\alpha$-AA seems relatively poor in vitro (Charles and Chang, 1981). Thus, high intracellular concentrations of $\alpha$-AA may be reached, causing osmotic and ionic disturbances, buildup of a toxic metabolic product, or block of a metabolic pathway in astrocytes. In the retina, glutamine synthetase activity (an enzyme considered to be selectively located in astrocytes; Norenberg and Martinez-Hernandez, 1979) has been reported to be reduced by $50 \%$ following a single intraocular injection of $\alpha$-AA in rats (Memo et al., 1981).

Among various disorders of the lysine metabolic pathway, $\alpha$ ketoadipic aciduria, which also causes the excretion of abnormal amounts of $\alpha$-AA, is particularly noteworthy here (Przyrembel et al., 1975). This defect of lysine metbolism is accompanied by an impairment of neuronal functions. Thus, our data concerning the gliotoxic effects of $\alpha$-AA (Huck et al., 1984) as well as the present study may become of interest also for neuropathologists.

\section{References}

Antanitus, D. S., B. H. Choi, and L. W. Lapham (1975) Immunofluorescence staining of astrocytes in vitro using antiserum to glial fibrillary acidic protein. Brain Res. 89: 363-367.

Balcar, V. J., and G. A. R. Johnston (1972) The structural specificity of the high affinity uptake of L-glutamate and L-aspartate by rat brain slices. J. Neurochem. 19: 26572666.

Balcar, V. J., J. Borg, and P. Mandel (1977) High affinity uptake of Lglutamate and L-aspartate by glial cells. J. Neurochem. 28: 87-93.

Bennet, J. P., W. J. Logan, and S. H. Snyder (1972) Amino acid neurotransmitter candidates: Sodium-dependent high-affinity uptake by unique synaptosomal fractions. Science 178: 997-999.

Bennet, J. P., W. J. Logan, and S. H. Snyder (1973) Amino acids as central nervous transmitters: The influence of ions, amino acid analogues, and ontogeny on transport systems for $\mathbf{L}$-glutamic and $\mathbf{L}$ aspartic acids and glycine into central nervous synaptosomes of the rat. J. Neurochem. 21: 1533-1550.

Casper, D. S., R. L. Trelstad, and L. Reif-Lehrer (1982) Glutamateinduced cellular injury in isolated chick embryo retina: Müller cell localization of initial effects. J. Comp. Neurol. 209: 79-90.

Chang, Y. F. (1978) Lysine metabolism in the rat brain: The pipecolic acid-forming pathway. J. Neurochem. 30: 347-354.

Chang, Y. F. (1982) Lysine metabolism in the human and the monkey: Demonstration of pipecolic acid formation in the brain and other organs. Neurochem. Res. 7: 577-588.

Charles, A. K., and Y. F. Chang (1981) Uptake, release, and metabolism of $\mathrm{D}$ - and $\mathrm{L}$ - $\alpha$-aminoadipate by rat cerebral cortex. J. Neurochem. 36: $1127-1136$.

de Barry, J., O. K. Langley, G. Vincendon, and G. Gombos (1982) LGlutamate and L-glutamine uptake in adult rat cerebellum: an autoradiographic study. Neuroscience 7: 1289-1297.

Drejer, J., O. M. Larsson, and A. Schousboe (1982) Characterization of L-glutamate uptake into and release from astrocytes and neurons cultured from different brain regions. Exp. Brain. Res. 47: 259-269.

Duce, I. R., P. L. Donaldson, and P. N. R. Usherwood (1983) Investigations into the mechanism of excitant amino acid cytotoxicity using a well-characterized glutamatergic system. Brain Res. 263: 77-87.

Eagle, H. (1955) Propagation in a fluid medium of a human epidermoid carcinoma, strain KB. Proc. Soc. Exp. Biol. Med. 89: 362-364.

Faivre-Bauman, A., J. Rossier, and P. Benda (1974) Glutamate accumulation by a clone of glial cells. Brain Res. 76: 371-375.

Gordon, R. D., and R. Balázs (1983) Characterization of separated cell types from the developing rat cerebellum: Transport of glutamate and aspartate by preparations enriched in Purkinje cells, granule neurones, and astrocytes. J. Neurochem. 40: 1090-1099.

Hatten, M. E., and R. L. Sidman (1978) Cell reassociation behavior and lectin-induced agglutination of embryonic mouse cells from different brain regions. Exp. Cell Res. 113: 111-125.

Hatten, M. E., A. M. Francois, E. Napolitano, and S. Roffler-Tarlov (1984) Embryonic cerebellar neurons accumulate $\left[{ }^{3} \mathrm{H}\right]-\gamma$-aminobutyric acid: Visualization of developing $\gamma$-aminobutyric acid-utilizing neurons in vitro and in vivo. J. Neurosci. 4: 1343-1353.

Henn, F. A., M. N. Goldstein, and A. Hamberger (1974) Uptake of the neurotransmitter candidate glutamate by glia. Nature 249: 663-664.

Huck, S. (1983) Serum-free medium for cultures of the postnatal mouse cerebellum: Only insulin is essential. Brain Res. Bull. 10:667-674.

Huck, S., and M. E. Hatten (1981) Developmental stage-specific changes in lectin binding to mouse cerebellar cells in vitro. J. Neurosci. 1: 1075-1084.

Huck, S., F. Grass, and M. E. Hatten (1984) Gliotoxic effects of $\alpha$ aminoadipic acid on monolayer cultures of dissociated postnatal mouse cerebellum. Neuroscience 12: 783-791.

Kizer, J. S., C. B. Nemeroff, and W. W. Youngblood (1978) Neurotoxic amino acids and structurally related analogs. Pharmacol. Rev. 29: 301-318.

Levi, G., G. P. Wilkin, M. T. Ciotti, and S. Johnstone (1983) Enrichment of differentiated, stellate astrocytes in cerebellar interneuron cultures as studied by GFAP immunofluorescence and autoradiographic uptake patterns with $\left[{ }^{3} \mathrm{H}\right] \mathbf{D}$-aspartate and $\left[{ }^{3} \mathrm{H}\right] \mathrm{GABA} . \mathrm{Dev}$. Brain Res. 10: 227-241.

Logan, W. J., and S. H. Snyder (1972) High affinity uptake systems for glycine, glutamic and aspartic acids in synaptosomes of rat central nervous tissues. Brain Res. 42: 413-431.

Memo, M., F. Riccardi, M. Trabucchi, and P. F. Spano (1981) Localization of GABA and dopamine receptor sites in retinal glial cells using DL- $\alpha$-aminoadipic acid. Adv. Biochem. Psychopharmacol. 26: $41-51$.

Neale, E. A., W. H. Oertel, L. M. Bowers, and V. K. Weise (1983) Glutamate decarboxylase immunoreactivity and $\gamma-\left[{ }^{3} \mathrm{H}\right]$ aminobutyric acid accumulation within the same neurons in dissociated cell cultures of cerebral cortex. J. Neurosci. 3: 376-382.

Norenberg, M. D., and A. Martinez-Hernandez (1979) Fine structural localization of glutamine synthetase in astrocytes of rat brain. Brain Res. 161: 303-310.

Nowak, L., P. Ascher, Y. Berwald-Netter, and F. Couraud (1983) Single channel currents of cultured mammalian astrocytes studied using excised membrane patches. Neurosci. Lett. Suppl. 14: S264.

Olney, J. W., O. L. Ho, and V. Rhee (1971) Cytotoxic effects of acidic and sulphur containing amino acids on the infant mouse central nervous system. Exp. Brain Res. 14: 61-76.

Olney, J. W., T. De Gubareff, and J. F. Collins (1980) Stereospecificity of the gliotoxic and anti-neurotoxic actions of alpha-amino-adipate. Neurosci. Lett. 19: 277-282.

Przyrembel, H., D. Bachmann, Y. Lombeck, K. Becker, U. Wendel, S. K. Wadman, and H. J. Bremer (1975) Alpha-ketoadipic aciduria, a new inborn error of lysine metabolism; biochemical studies. Clin. Chim. Acta 58: 257-269.

Puil, E. (1981) S-Glutamate: Its interactions with spinal neurons. Brain Res. Rev. 3: 229-322.

Reiser, G., F. Löffler, and B. Hamprecht (1983) Tetrodotoxin-sensitive ion channels characterized in glial and neuronal cells from rat brain. Brain Res. 261: 335-340.

Roberts, P. J., and J. C. Watkins (1975) Structural requirements for the inhibition for L-glutamate uptake by glia and nerve endings. Brain Res. 85: 120-125.

Schmid, R., J. S. Hong, J. Meek, and E. Costa (1980) The effect of kainic acid on the hippocampal content of putative transmitter amino acids. Brain Res. 200: 355-362.

Schousboe, A., G. Svenneby, and L. Hertz (1977) Uptake and metabolism of glutamate in astrocytes cultured from dissociated mouse brain hemispheres. J. Neurochem. 29: 999-1005.

Stallcup, W. B., K. Bulloch, and E. E. Baetge (1979) Coupled transport of glutamate and sodium in a cerebellar nerve cell line. J. Neurochem. 32: 57-65.

Trenkner, E., and R. L. Sidman (1977) Histogenesis of mouse cerebellum in microwell cultures. Cell reaggregation and migration, fiber and synapse formation. J. Cell Biol. 75: 915-940. 
Vincent, S. R., and E. G. McGeer (1980) A comparison of sodiumdependent glutamate binding with high-affinity glutamate uptake in rat striatum. Brain Res. 184: 99-108.

Wheeler, D. D. (1979) A model of high affinity glutamic acid transport by rat cortical synaptosomes-a refinement of the originally proposed model. J. Neurochem. 33: 883-894.
White, W. F., S. R. Snodgrass, and M. Dichter (1980) Identification of GABA neurons in rat cortical cultures by GABA uptake autoradiography. Brain Res. 190: 139-152.

Wilkin, G. P., J. Garthwaite, and R. Balázs (1982) Putative acidic amino acid transmitters in the cerebellum. II. Electron microscopic localization of transport sites. Brain Res. 244: 69-80. 